\title{
Design of LAN Platform for the Fusion of Communication and Networking
}

\author{
LI Busheng ${ }^{1, a}$, HU Jingfang, ${ }^{1, b}$ \\ ${ }^{1}$ School of Information Engineering, Jingdezhen Ceramic Institute, \\ Jiangxi, P.R. China

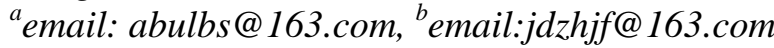

\begin{abstract}
LAN Platform is application software based on UDP for LAN chat software, set the mainstream communication functions into one body, play LAN stability, quick advantage, provide interactive communication platform for LAN users. The software does not require a server, start the engine and the current online users, the software also provides cross network game search function, provides for the majority of War3 lovers convenient battle platform.

Keyword: LAN Platform; UDP ; TCP ; WarCraft3; LAN
\end{abstract}

\section{Introduction}

In our daily computer use, through the network communication is very necessary. Instant communication within the LAN has been occupied by the Dove Chuanshu software, during the period of working in many domestic companies are not allowed to log on QQ communication software, so the Dove Chuanshu LAN become everybody based selection, had to admit the Dove Chuanshu is a classic small software, but after all the time already a long time of the functionality it offers far can not meet the needs of contemporary people.

Along with the network development of the battle platform becomes hot, Haofang, VS, GG etc. are numerous, but limited to the conditions of access to the Internet, speed restriction, smooth game effect not to many internal users, is lag trouble. Within the LAN network can almost be ignored the concept of lag, but many games can only be in the same subnet online, the network also spread relevant LAN cross segment on-line software such as: Lancraft, BattleLAN[2], Game Searcher etc.. But these software and less convenient battle platform, need to contact well determine the host can be used, and some software also exists bug.

In this paper, the design of software to messaging and online software function are combined to use in the LAN, can exchange can also resolve the online lag issue, namely the battle platform simple, local area network . 


\section{Key technology analysis}

LAN Platform is a socket based network programming work, for the majority of network users, compared with other communication software, the biggest feature is detached from the server without bound, the login process cumbersome, open the can communicate with others. Greatly enhance the convenience of the software [5]. The software adopts the standard socket development, applicable to a variety of local area network environment; improve the software compatibility and stability, and the Windows operating system on the various versions of the good support.

Socket originally comes from UNIX, is the network communication interface of UNIX operating system, Berkeley for the development of University of California [1]. With the wide use of UNIX operating system, Socket is also fully deserve to be one of the most popular network communication program interface. In early 90, by SUN Microsystems, JSB.CO, FTP Software, Microdyne and Microsoft and other companies jointly developed a standard socket programming a set of Windows, called the Windows Sockets specification, the initial realization of Sockets interface program under Windows standard, and in 1992 launched WindowsSocketsV1.0 version, the following year published its 2 version IBM release of the TCP/IPV2.1forDOS is its representative, it also provides application program interface Windows Sockets (API). Microsoft Windows Sockets API is a network application program interface under Windows, in order to message mechanism and induction for Windows under the I/O Windows Sockets API selection operation, expansion of nearly 20 functions in function, which extended part are preceded by the prefix WSA (Windows Sockets Asynchronous), such as WSAStartup, WSA Clean etc, fully embody the superiority of Windows.

Socket (Socket) can be regarded as an endpoint for communication connection in two procedures, a program, a message will be written into the Socket; this information will be sent to another Socket, so that this information can be transmitted to other programs.

\section{2 software analysis}

\subsection{Analysis of software objects}

The development orientation of the software for LAN, without the need for external network authentication, can with LAN other user interaction, transfer files, searches for LAN war3 host to achieve LAN simple battle platform [3]. For the enterprise's internal network, not allowed to log on QQ, MSN and other IM software, you can use the software, transmission of communication and resource sharing. Software integration to remind function can make the reminder of busy you promptly, so as not to delay the work of learning, reminder software in daily use to make it more intimate and 
more humane. The design goals with include:

1) Establish a for LAN communication platform, information exchange, file sharing service.

2) To realize the acquisition and display of online users, chat with the specified user interaction, expression, chat, send content screen processing window jitter function.

3) To achieve the right click menu message response, shear, replication, revocation and other commonly used command, send the font properties of individual settings, and open the saved chat record.

4) Implementation of file transfer function is sent to the specified users, select and monitors the status of file transfer and prompt result report.

5) To provide WarCraft3 LAN game host search function, including the subnet search segments settings, WarCraft3 version of the set and search condition monitoring.

6) Analysis the WarCraft3 returns data packet and will host the information displayed in the user interface, in addition to achieve the specified calling directly to the external program WarCraft3 includes the selection and operation parameters of the running path.

7) The realization of personal information set, including head choice and personal name, nickname, QQ, telephone, home page, E-Mail, character signature information.

8) Provide a simple reminder function, including the event theme, event time setting, voice prompts, events specified content description. To provide event reminders, the whole point timekeeping function of state control, that is, whether to open.

9) Implementation of the chat page window jitter and information prompt sound state control, i.e., whether to open. To provide a network of users add chat neutron namely send subnet search news.

10) To provide the help file and tips on the open button, convenient for the user to refer to the instructions for use, method of using master the use of the software users quickly.

11) Preserving all settings, saved to the corresponding position of the win.ini file and registry.

\subsection{Analysis of software function}

Construction of software based on the MFC dialog box, consists of four main functions, respectively is: function, game function, the function of personal information and setting function chat.

Chat function is the main function of the software, the default display software startup function. Chat on instant communication mainly, offer online list, select the user display, right-click menu, set the font properties in response, open the chat record, expression selector, and window jitter, file transfer and other functions.

The game features is another function of the software, mainly on the local area network WarCraft3 game host to search and display the host information map information content. Provide other sub network search 
settings, WarCraft3 version of the game, the game path and operation parameter settings, start the game etc..

User settings related information of individuals for personal information function, so as to display more information to other users in the online list. Can be set to include: head, nicknames, real name, sex, QQ, MSN, phone, E-Mail, dormitory, VSID, personal homepage, character signature, on-line greeting etc.

Set the function provides the related settings of the software and the function of reminding. Can control the window jitter, chat in the message tone is opened and closed, to other subnets need to chat users IP settings. Reminding function setting and the whole point timekeeping to open and close. In addition, this function can also open a help file and the daily post function set.

\section{The software design}

\subsection{The relationship between the modules}

Including chat, games, personal information and system function setting of four main function modules in the main frame of the software, each function module is attached to the main frame, personal information module and system function setting module provides support for the chat module, system settings module and function module provide support to the game, the relationship of each module as shown in Figure 2 shows.

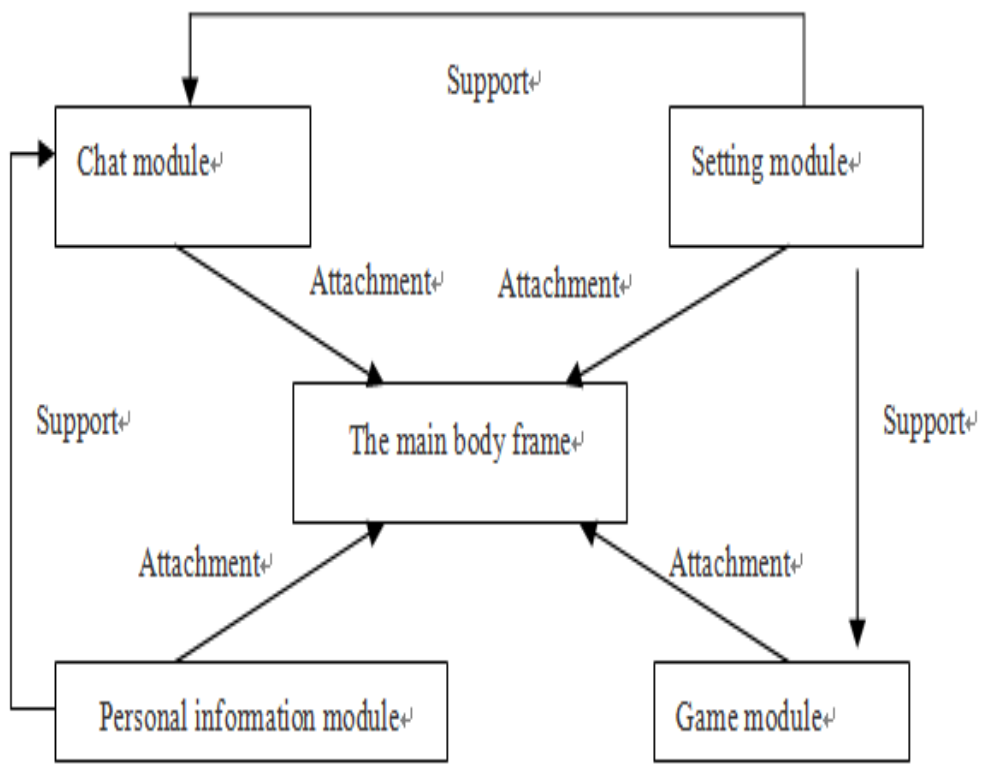

Fig. 1 Relation picture among every sub page 


\subsection{Business flow chart of the main module}

The flow chart of the business chat module as shown in figure 2.

\section{Realization of the system}

The software uses Microsoft Visual C++ 6 as the main development tools [4], to achieve the following four functions: chat, chat implementation of LAN based on UDP TCP file transfer function based on cross network segment; games, WarCraft3 games to achieve the search function, the application of the network packet capture, packet analysis technology; personal information for user settings personal information, providing more information for the chat function; system settings, for related content, set the system such as: open state control timing reminder, the whole point timekeeping, news voice prompts, daily post, help files and other functions.

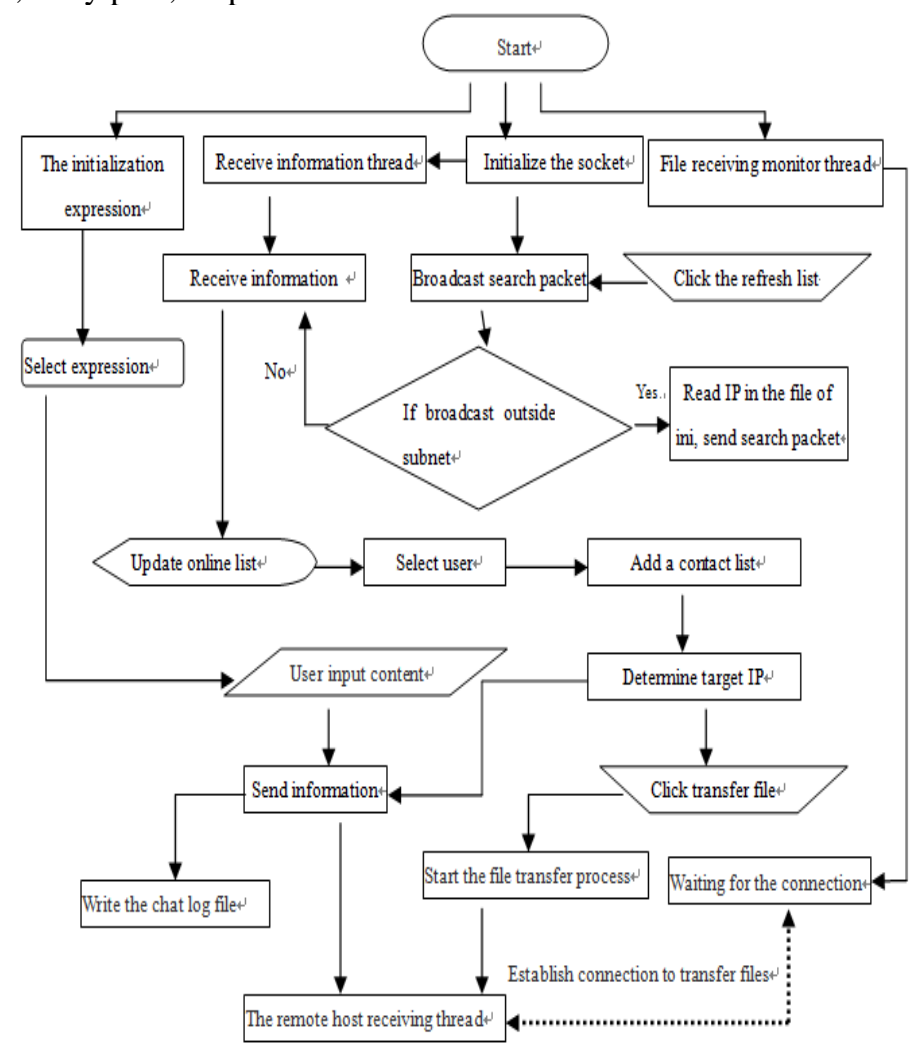

Fig.2 Chat page flow chart 


\section{Conclusions}

LAN chat tool is used for communication between the internal LAN users a tool, generally have a text chat and file transfer functions. It has less consumption of system resource, the advantages of faster. In this paper, the development of the software also provides cross network game search function, provides for the majority of War3 lovers convenient battle platform.

\section{Reference}

[1] Sun Haimin. Proficient in Windows Sockets network development - Visual $\mathrm{C}++$ based on the realization of the posts and Telecommunications Press.2008.7.

[2] Zhang Yong. Analysis of Teaching LAN security. The network security technology and application of [J].2010-3.

[3] James F.Kurose. Computer Networking-A Top-Down Featuring the Internet [M]. The posts and Telecommunications Press.2004.1.

[4] B. Ford, M.I.T., Srisuresh, etc. Peer-to-Peer (P2P) communication across middle boxes. http://midcom-p2p.sourceforge.net/draft-ford-midcom-p2p-0 1.txt 2014

[5] Isabella Kamau. Platform. Raider Publishing International.2009.6 